\title{
Twist is required for viability, migration, invasion and chemoresistance of the MDA-231LM3.3 triple-negative breast cancer cells
}

Corresponding author:

Tao $\mathrm{He}$

Institute for Cancer Medicine, Sichuan Medical University,3-319 Zhongshan Road, Jiangyang District, Luzhou, Sichuan 646000, China.

E-mail: hetao198@163.com.

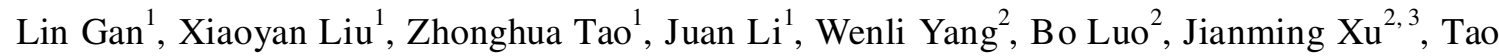
$\mathrm{He}^{2, *}$

1. The Research Center for Preclinical Medicine, Sichuan Medical University, Luzhou, Sichuan, China.

2. Institute for Cancer Medicine and College of Basic Medical Sciences, Sichuan Medical University, Luzhou, Sichuan, China.

3. Department of Molecular and Cellular Biology, Baylor College of Medicine, Houston, TX, USA

Running title: Twist in MDA-231LM3.3 cells

\begin{abstract}
Most triple-negative breast cancers (TNBCs) are highly malignant and resistant to chemotherapy. Because TNBCs do not express estrogen receptor (ER ) and human epidermal growth factor receptor 2 (HER2), the currently available therapies that target estrogen and HER2 signaling pathways are not applicable for TNBC treatment. Twist is frequently expressed in TNBC cells and it has been shown to promote epithelial mesenchymal transition (EMT), migration, invasion, metastasis and survival of many types of cancer cells. To define the specific role of Twist in TNBC cells, we generated MDA-231LM3.3 cells expressing either non-targeting shRNAs or Twist mRNA-targeting shRNAs and analyzed the effects of Twist silencing on the viability, behavior and chemoresistance of these cells. We found that knockdown of Twist in MDA-231LM3.3 TNBC cells significantly decreased their colony formation capability and increased their apoptosis rate. Furthermore, knockdown of Twist in these cells also significantly inhibited their migration and invasion capabilities and elevated their sensitivities to breast cancer chemotherapeutic drugs including Cisplatin, Docorubicin and Vinorelbine. These results indicate that Twist is required for the viability, migration, invasion and chemoresistance of MDA-231LM3.3 cells, suggesting that Twist may serve as a molecular target for controlling certain TNBCS.
\end{abstract}


Twist is required for viability, migration, invasion and chemoresistance of the

MDA-231LM3.3 triple-negative breast cancer cells

\section{Introduction}

About $15 \%$ of all breast cancer cases belong to triple-negative breast cancer (TNBC), which expresses no estrogen receptor (ER ) and progesterone receptor (PR) and a little human epidermal growth factor receptor 2 (HER2). Therefore, the currently available endocrine therapy that targets the estrogensignaling pathway and the targeted therapy that inhibits HER2 are not applicable to treat TNBC [1, 2]. Since TNBC is associated with high risks of recurrence and metastasis, poor responses to chemotherapy, and short overall survival $[1,2]$, it is important to identify new molecular targets for developing new therapeutic drugs for treating TNBC.

Twist is a highly conserved basic helix-loop-helix (bHLH) transcription factor that can form heterodimers with E12 or E47 to activate or inhibits different target gene expression. Twist plays a pivotal role in a number of essential developmental programs, such as mesoderm induction and differentiation [3]. Furthermore, Twist is usually not expressed in normal epithelial cells, but its expression is induced in many types of cancer cells such as certain breast, hepatocellular, prostate, gastric and pancreatic cancer cells [3]. Twist plays an important role in promoting epithelialmesenchymal transition (EMT), migration, invasion and metastasis of cancer cells and in increasing cancer stem cell population and cancer cell survival and resistance to apoptosisinducing reagents [3-6].
In breast cancer researches, Twist was initially identified to be required for lung metastasis of breast cancer cell-derived xenograft tumors in mice [4]. Subsequently, Twist was found to convert MCF7 epithelial breast tumor cells into mesenchymal like tumor cells, and these Twist-overexpressing MCF7 cells showed enhanced viability and chemoresistance [5, 6]. It was also demonstrated that Twist expressed in both tumor cells and endothelial cells promoted tumor angiogenesis [7, 8] and that Twist expressed in tumor cells re-programed these cell's trans-differentiation into tumor endothelial cells [9]. At the molecular level, Twist is stabilized by MAPK-mediated phosphorylation [10]. Twist interacts with NuRD complex to repress E-cadherin and ER expression, which promotes EMT and the invasive capability of breast cancer cells [11, 12]. In addition, Twist can also upregulate Bmi1 to promote EMT and interacts with BRD4 to activate gene expression and facilitate basal-like breast tumorigenesis [13, 14]. In summary, these findings suggest that Twist is a detrimental transcription factor that actively promotes breast cancer progression and metastasis.

In this study, we further investigated the role of Twist in a recently established MDA-231LM3.3 cell line, which is a highly metastatic TNBC cell line. We report that Twist is required for the viability, migration, invasion and chemotherapeutic resistance of the MDA-231LM3.3 TNBC cells. 
Twist is required for viability, migration, invasion and chemoresistance of the

\section{Materials and Methods}

Cell culture and lentrinal shRNA based knockdown of gene expression

The human MDA-231LM3.3 triplenegative breast cancer (TNBC) cell line was previously described [15]. This cell line was maintained in DMEM containing $10 \%$ fetal bovine serum (FBS). HEK293T cells were transfected with GIPZ lentiviral vectors for expressing non-targeting control shRNAs or Twist mRNA-targeting shRNAs (Open Biosystems). The produced lentiviral particles in the conditioned medium were used to infect MDA-231LM3.3 cells as described previously [11]. The infected cells were cultured in the growthselection medium containing $1 \mathrm{ug} / \mathrm{ml}$ of puromycin for 5 days to establish stable cellular pools for experiments.

\section{RNA extraction and RT-PCR analysis}

Total RNA samples were extracted from MDA-231LM3.3 cells expressing non-targeting shRNAs or Twist-targeting shRNAs, followed by reverse transcription using a reverse transcriptase kit (Invitrogen) as described previously [16, 17]. PCR was performed with $1 \mathrm{ug}$ of cDNA and a pair of Twist-specific primers (5'-acgagctggactccaagatg and 5'cacgccetgtttctttgaat) or a pair of GAPDH-specific primers (5'ccatgttcgtcatgggtgtgaacca and 5 'gccagtagaggcagggatgatgttc). PCR products were separated in $1.5 \%$ agarose gel by electrophoresis.

\section{Western blotting analysis}

Western blotting analysis was performed as described previously [10, 18]. Briefly, whole-cell lysate was prepared and protein concentration was assayed by using the BCA protein assay reagent. A total of $35 \mathrm{ug}$ protein in each cell lysate was separated by electrophoresis in
$10 \%$ SDS-PAGE gels, transferred onto PVDF membranes, and probed with Twist antibody (Abcam, 1:1000 dilution) and GAPDH antibody (Cell Signaling Technology, 1:5000 dilution). Then, the membranes were incubated with a horseradish peroxidase-conjugated secondary antibody, followed by visualizing the signals by reacting to the Western Blot Chemiluminescent Substrate (Pierce, USA) and exposing to X-ray films.

\section{Cell Counting Kit-8 assay}

Total viable cells were assayed by using the Cell Counting Kit-8 (CCK8) according to the manufactural instruction (Dojindo, Kumamoto, Japan). Briefly, MDA-231LM3.3 cells with either control shRNA or Twist mRNA-targeting shRNA expression were plated into 96-well plate $\left(4 \times 10^{3}\right.$ cells/well $)$ and cultured for 24 hours. The CCK8 reagent was added into each well for 1 hour at $37^{\circ} \mathrm{C}$. The absorbance (optical density) at $450 \mathrm{~nm}$ was measured with a plate reader

(Multiskan GO Microplate

Spectrophotometer, Thermo Fisher

Scientific, USA). All experiments were repeated three times.

\section{Cell colony formation assay}

MDA-231LM3.3 cells expressing control or Twist mRNA-targeting shRNAs were culture in 6-well plates in triplicate at a seeding density of 300 cells/well. Culture medium (DMEM with $10 \%$ FBS) was changed every 3 days. After cultured for 14 days, the formed cell colonies were fixed with $70 \%$ methanol and stained with $0.5 \%$ crystal violet containing 20\% methanol for 15 minutes. The stained Colonies consisting of $>50$ cells were counted under a microscope. All experiments were performed in triplicate and the average number of colonies in each group was presented. 
Twist is required for viability, migration, invasion and chemoresistance of the

MDA-231LM3.3 triple-negative breast cancer cells

\section{fACS analysis}

Cells were seeded in six-well plate $(5 \times 105$ cells/well $)$ and cultured in DMEM medium supplemented with $10 \%$ FBS for 24 hours. Cells were harvested, washed twice with cold phosphate-buffered saline (PBS), fixed in $70 \%$ ethanol, stained with propidium iodide $(10 \mu \mathrm{g} / \mathrm{ml})$, and subjected to FACS analysis. The cell fractions in $\mathrm{G}_{0} / \mathrm{G}_{1}, \quad \mathrm{~S}$, and $\mathrm{G}_{2} / \mathrm{M}$ phases were determined using ModiFIT software (Becton Dickinson). All samples were analyzed using a Becton Dickinson FACS Calibur flow cytometer. All experiments were performed in triplicate.

\section{Cell apoptosis assay}

Cells were seeded at $5 \times 10^{5}$ cells/well in triplicate in six-well plate and cultured for 24 hours. Cells were washed twice with cold PBS, and stained using Annexin V and propidium iodide according to the manufacturer's instructions. The stained cells were assessed by flow cytometry using CellQuest software.

\section{Cell migration and invasion assays}

Cell migration and invasion assays were performed in Corning Trans-well migration and Matrigel-coated invasion chambers (Corning, USA, NY) as described previously [19]. Briefly, $2.5 \times 10^{4}$ of MDA-231LM3.3 cells with normal or knockdown of Twist expression were seeded in each upper chamber of the trans-well chamber containing $100 \mathrm{ul}$ of serum-free DMEM medium. The lower chamber was filled with DMEM containing $10 \%$ FBS. After culturing for 24 hours, the non-migrating/non-invading cells retained in the upper chambers were removed from the membranes with a cotton swab, and the migrated/invaded cells in the lower chambers, which attached to the reverse side of the membranes, were fixed, stained with $0.1 \%$ violet crystal dye and counted in five randomly selected fields $(100 \times)$ under a phase contrast microscope. Each experiment was performed in triplicate. The stained cells were washed with $33 \%$ acetic acid and the extracted dye from the stained cells was measured by using a Microplate Reader (Thermo, Mulitskan Go) at OD570 $\mathrm{nm}$.

\section{Cisplatin, Doxorubicin, and Vinorelbine Treatments}

\section{MDA-231LM3.3 cells expressing control or Twist mRNA-targeting shRNAs were seeded at a density of $5.0 \times 10^{3}$ cells/well in six-well plate. After cultured overnight, the cells were treated with different concentrations of Cisplatin (DDP), Doxorubicin (ADM), or Vinorelbine (NVB). After 24 hours of treatment, the CCK8 reagent was added into each well for 1 hour at $37^{\circ} \mathrm{C}$. The absorbance (optical density) at $450 \mathrm{~nm}$ was measured with a plate reader. All experiments were repeated in triplicate.}

\section{Statistical analysis}

Data obtained from three independent experiments were presented as Mean \pm standard deviation (SD). These data were statistical analyzed by One-way ANOVA, followed by a Student-Newman-Keuls multiple comparison test. A $P$ value $<0.05$ was considered significantly different.

\section{Results}

\section{Silencing Twist expression in MDA-} 231LM3.3 cells decreased cell growth in number and colony formation capability

The MDA-231LM3.3 cell line is a human TNBC cell line that develops extensive lung metastasis when orthotopically inoculated in the mammary fat pads of immune-defect mice [15]. To investigate the role of Twist in these TNBC cells, we employed the GIPZ Lentiviral shTwistmir-GFP

(Open 
Twist is required for viability, migration, invasion and chemoresistance of the

Biosystems) to knock down Twist expression. Lentiviruses that co-express GFP with Twist shRNAs (shTwist) or with non-targeting shRNAs (control) were used to infect MDA-231LM3.3 cells. In the resulted cellular pools, both Twist mRNA and protein were significantly knocked down by Twist shRNAs when compared with non-targeting shRNAs (Figure $1 \mathrm{~A}$ and B). To determine the effect of Twist knockdown on cell viability, we performed Cell Count Kit-8 (CCK-8) assay to compare cell number growth between MDA-231LM3.3 cells with and without Twist knockdown. We found that MDA231LM3.3 cells with Twist knockdown showed a significantly lower growth in cell number compared with the control MDA-231LM3.3 cells without Twist knockdown (Figure 2), which could be a consequence of either decreased cell proliferation or increased cell death.

Next, we further examined the effect of Twist knockdown in MDA-231LM3.3 cells on colony formation under a very low cell density setting. The number of colonies $(18.07 \pm 1.31)$ formed from MDA-231LM3.3 cells with Twist knockdown was significantly lesser than the number of colonies $(27.83 \pm 3.12)$ formed from the control cells with normal Twist expression (Figure 3). These results indicate that Twist is required for maintaining the colony formation capability in MDA-231LM3.3 cells.

A
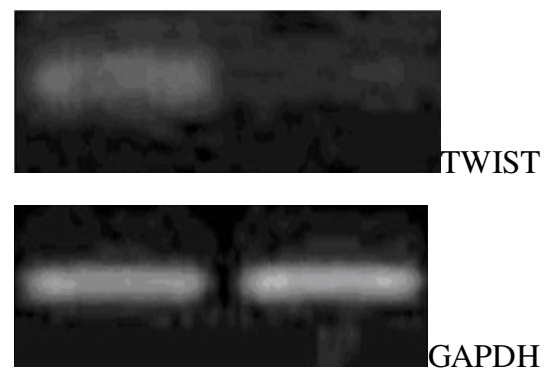

Con

shTwist

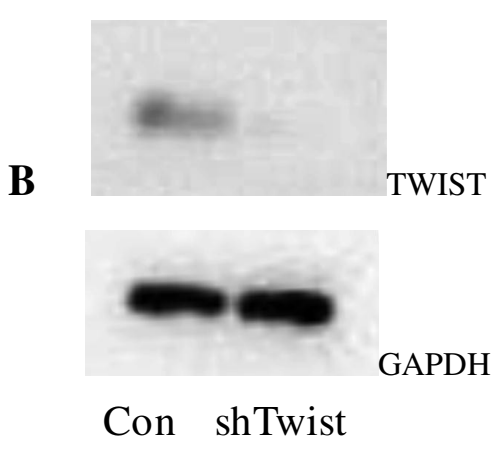

Figure 1. Knockdown of Twist mRNA and protein in MDA-231LM3.3 cells. RT-PCR analysis of Twist mRNA expression (Panel A) and Western blot analysis of Twist protein expression (Panel B) in MDA-231LM3.3 cells expressing control (Con) non-targeting shRNA and Twist mRNA-targeting shRNA (shTwist).

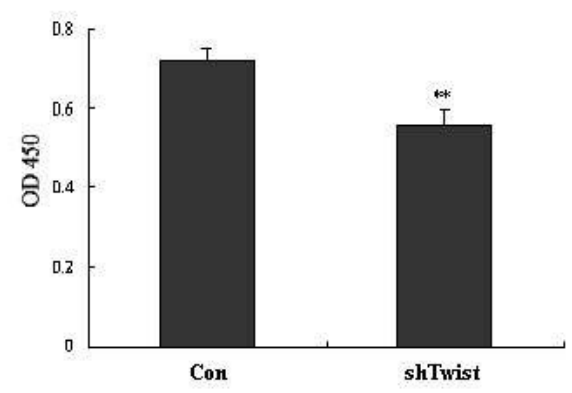

Figure 2. Knockdown of Twist in MDA-231LM3.3 cells decreased cell number. MDA-231LM3.3 cells expressing non-targeting shRNAs (Con) or Twist mRNA-targeting shRNAs were cultured for 24 hours. The relative cell numbers were assessed by CCK- 8 assay. The data from three independent experiments are presented as Mean \pm SD. $* *, P<0.01$.

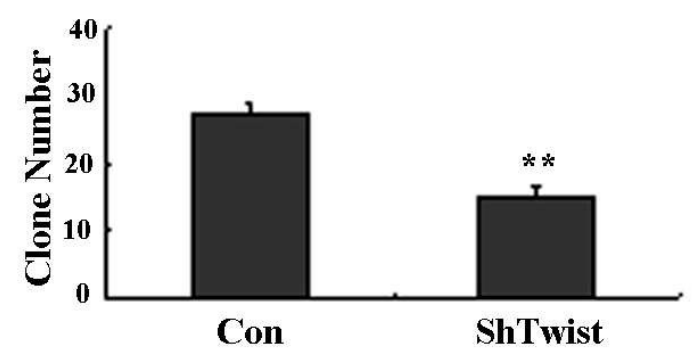


Twist is required for viability, migration, invasion and chemoresistance of the

MDA-231LM3.3 triple-negative breast cancer cells

Figure 3. Knockdown of Twist in MDA-231LM3.3 cells reduced colony formation. MDA-231LM3.3 cells expressing control (Con) or Twist mRNA-targeting shRNAs were cultured at very low density for 14 days. The formed cell colonies were stained and counted. Data obtained from three independent experiments are presented as Mean \pm SD. $\quad * *, P<0.01$.

Knockdown of Twist expression in MDA231LM3.3 cells increased both cell proliferation and apoptosis

To assess the cellular mechanisms responsible for the decreased growth in cell number and

decreased colony formation caused by Twist knockdown, we first performed FACS assay to examine the effect of Twist knockdown on cell cycle progression. When compared with control MDA231LM3.3 cells with normal Twist expression, the G1-phase fraction of MDA231LM3.3 cells with Twist knockdown was reduced, while their S-phase and G2-phase fractions were elevated (Figure
$4 \mathrm{~A}$ and $\mathrm{B})$. These results suggest that knockdown of Twist in MDA-231LM3.3 cells did not slow down cell cycle, but actually accelerated cell cycle. Thus, the decreased cell number and colony formation induced by Twist knockdown was not caused by any decrease in cell proliferation.

We also examined the effect of Twist knockdown on cell apoptosis induced by culturing cells in serum-free medium for 24 hours. The percentage (12.07 \pm $1.01 \%)$ of apoptotic

MDA-231LM3.3 cells with Twist knockdown was much higher than the percentage $(6.68 \pm$

$0.28 \%$ ) of apoptotic control MDA231LM3.3 cells (Figure 5A and B). These results suggest that Twist is required for maintaining the normal survival of MDA231LM3.3 cells. Thus, the decreased cell number and colony formation induced by Twist knockdown is likely attributed to the decreased cell viability caused by Twist silencing.
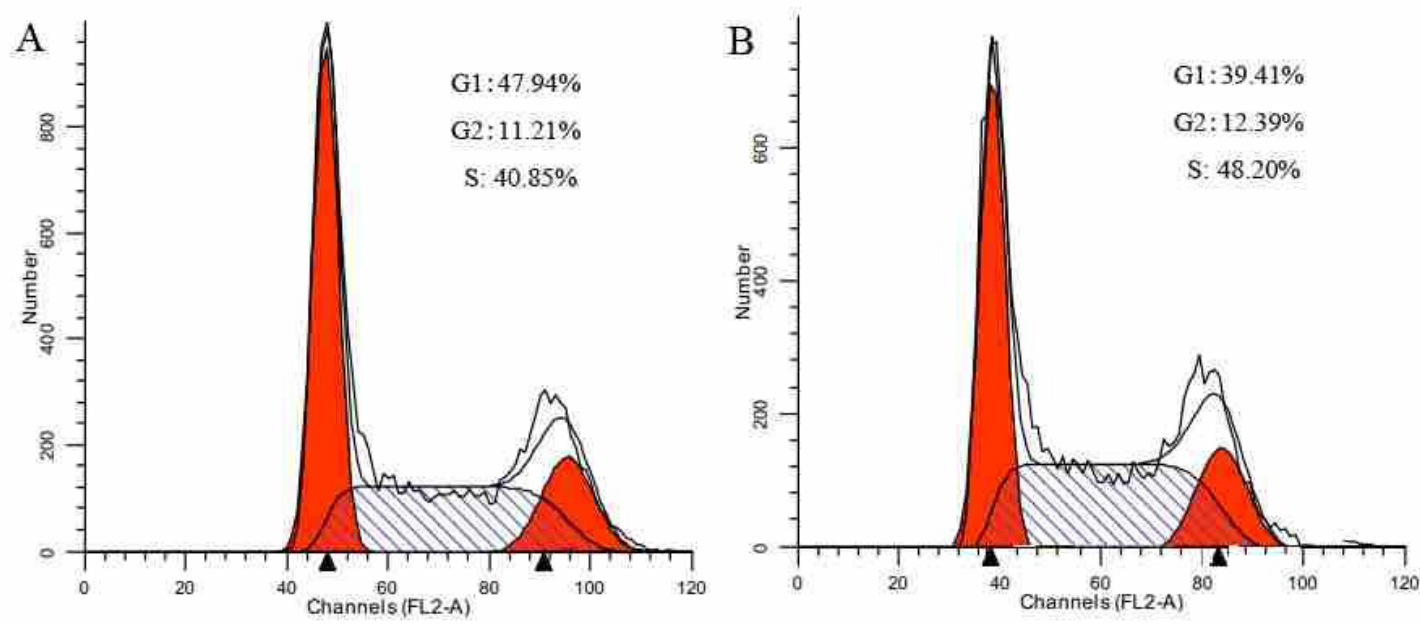

Figure 4. Knockdown of Twist in MDA-231LM3.3 cells altered cell cycle. Cultured MDA-231LM3.3 cells with control (Con) shRNA expression (Panel A) or Twist mRNA-targeting shRNA expression (Panel B) were analyzed by FACS. Cell fractions in G1, S, and $\mathrm{G} 2 / \mathrm{M}$ phases are indicated. 


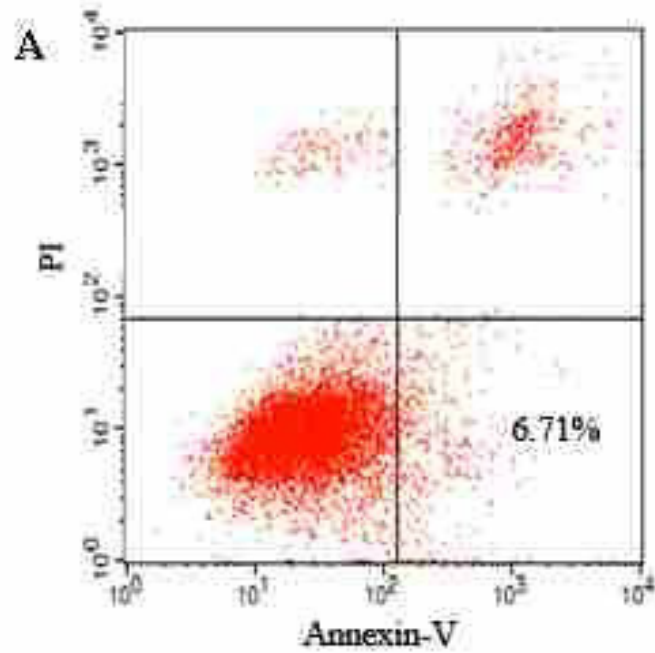

Figure 5. Knockdown of Twist increased the apoptosis of MDA-231LM3.3 cells.

MDA-231LM3.3 cells with control shRNA expression (Panel A) or Twist mRNA-targeting shRNA expression (Panel B) were stained with fluorescence labeled Annexin $\mathrm{V}$ and propidium iodide and analyzed by flow cytometry. The percentages of apoptotic cells indicated in the scatter plots were calculated from three repeat assays.

Knockdown of Twist inhibited the migration and invasion capabilities of MDA-231LM3.3 cells

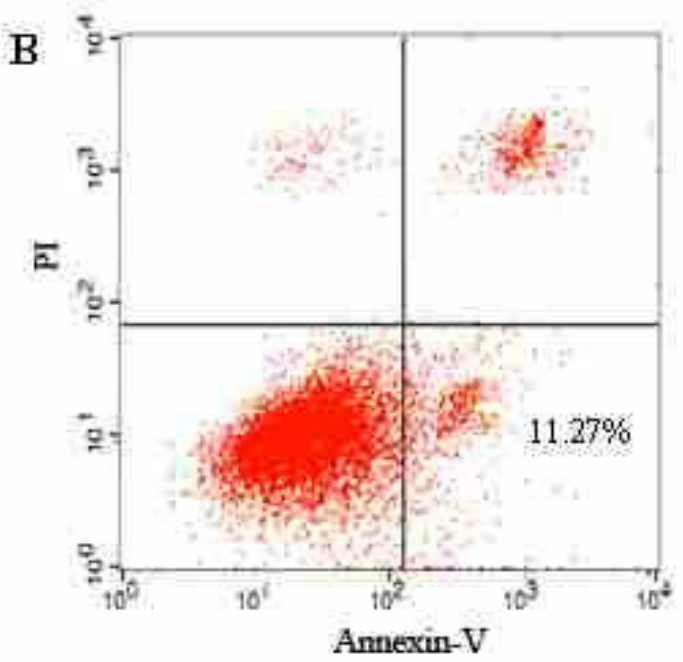

To examine the role of Twist in migration and invasion of MDA231LM3.3 cells, we performed transwell assays to compare the migration and invasion capabilities of MDA-231LM3.3 cells with normal Twist expression and Twist knockdown. These assays revealed that knockdown of Twist in MDA231LM3.3 cells significantly reduced cell migration and invasion by about $40 \%$ and $50 \%$, respectively (Figure 6A-C). These results indicate that Twist is required for maintaining the aggressive migration and invasion capabilities of these TNBC cells. 


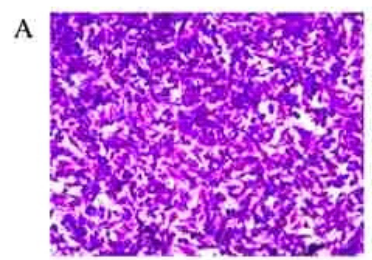

Con

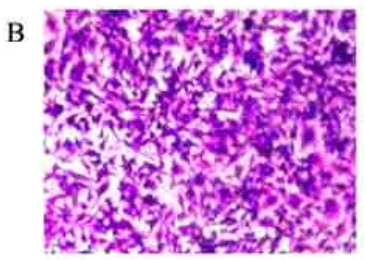

Con

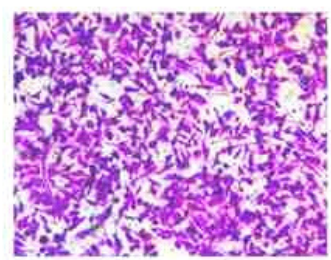

shTwist

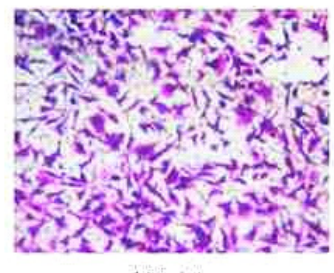

shTwist
Figure 6. Knockdown of Twist repressed the migration and invasion of MDA231LM3.3 cells. A. Photographs of migrated MDA-231LM3.3 cells with control shRNA expression (Con) or Twist mRNA-targeting shRNA expression (shTwist). The images were taken under a microscope with 200x magnification. B. Images of invading MDA-231LM3.3 cells with control shRNA expression (Con) or Twist mRNA-targeting shRNA expression (shTwist), which were recorded under a microscope with 200x magnification. C. The average numbers of migrated and invaded MDA-231LM3.3 cells with control shRNA expression (Con) or Twist $m R N A$-targeting shRNA expression (shTwist). Data were obtained from three independent assays and statistically analyzed by Student's $t$ test. **, $P<0.01$.

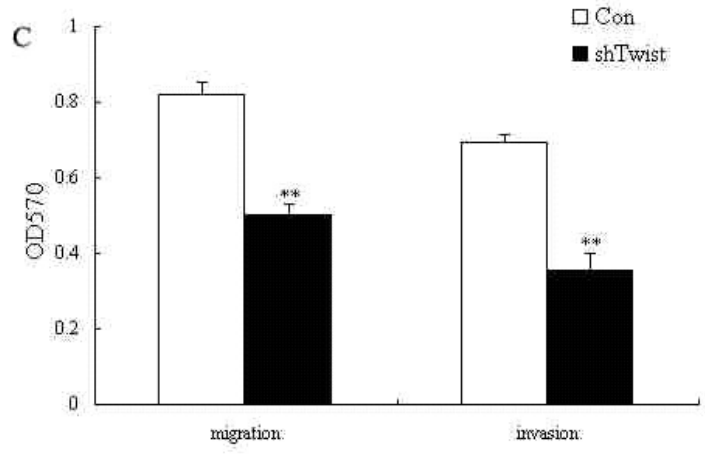

\section{Knockdown of Twist expression enhanced the sensitivity of MDA- 231LM3.3 cells to chemotherapy}

To examine the role of Twist in determining the sensitivity of TNBC cells to chemotherapy, we treated MDA231LM3.3 cells with normal or silenced Twist expression with Cisplatin (DDP), Doxorubicin (ADM), or Vinorelbine $\mathrm{ug} / \mathrm{ml}$, the EC50 of ADM from 16 $\mathrm{ug} / \mathrm{ml}$ to $8 \mathrm{ug} / \mathrm{ml}$, and the EC50 of NVB from $20 \mathrm{ug} / \mathrm{ml}$ to $12 \mathrm{ug} / \mathrm{ml}$ (Figure 7A-C). These results clearly demonstrated that knockdown of Twist in MDA-231LM3.3 cells significantly reduces the resistance of these TNBC cells to chemotherapeutic drugs.

(NVB). After treated for 24 hours, cell viability assays

revealed that knockdown of Twist significantly decreased the EC50 of DDP from $10 \mathrm{ug} / \mathrm{ml}$ to 7.5 

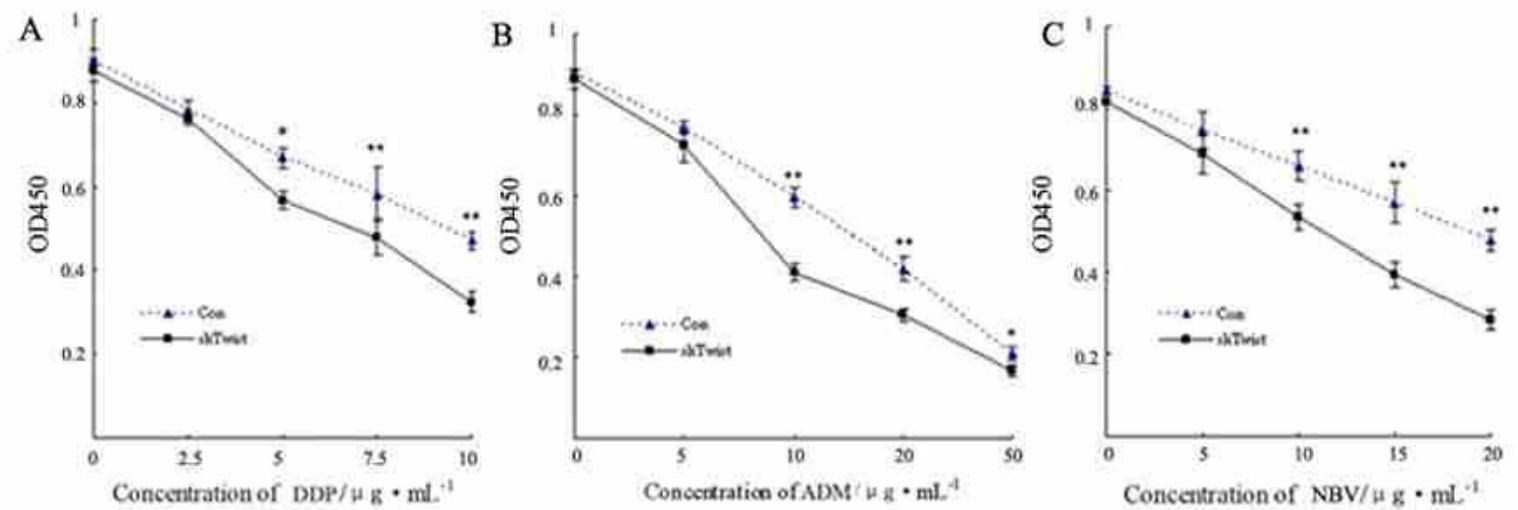

Figure 7. Knockdown of Twist increased the inhibitory effects of DDP, ADM and NVB on MDA231LM3.3 cell growth. MDA231LM3.3 cells with control shRNA expression (dotted lines) or Twist mRNA-targeting shRNA expression (solid lines) were treated with DDP, $\mathrm{ADM}$ or NVB at different concentrations as indicated for 24 hours. The relative numbers of viable cells in each treatment group were measured by CCK- 8 assays. The data obtained from three independent assays are presented as Mean \pm SD and statistically analyzed by Student's t test. ${ }^{*}, P<0.05 ; * *, P<0.01$.

\section{Discussion}

Twist is one of the master transcription factors that drive EMT, migration and invasion of various types of cancer cells [3]. Although its roles in converting epithelium-like breast tumor cells into fibroblast-like invasive breast cancer cells and in promoting migration, invasion and metastasis of the mouse ER -negative breast tumor cells and the MDA-MB-435 human melanoma cells have been extensively investigated $[4,11$, $12,17,20]$, the role of Twist in human TNBC cells has not been fully understood. In this study, we found that knockdown of Twist expression in MDA-231LM3.3 cells significantly decreased the capabilities of these cells to form colonies under a low cell density setting, to migrate on the culture plate and invade through a layer of Matrigel, and to survive against chemotherapeutic drugs. Collectively, these results demonstrate that the basic role of Twist in MDA231LM3.3 cells are consistent with the role previously defined in most other types of cancer cells, which is to promote cell migration, invasion, survival and chemoresistance [3]. Therefore, these results also suggest that Twist may serve as a molecular target in TNBC cells such as MDA-231LM3.3 cells for controlling these cancer cell viability and invasive behavior.

Interestingly, we found that knockdown of Twist expression in MDA231LM3.3 cells decreased the number of total cells in the culture, which was caused by increased apoptosis and decreased cell viability as indicated by the reduced colony formation, but not by decreased cell proliferation. Actually, knockdown of Twist slightly increased the proliferation of MDA-231LM3.3 cells, which was a different result from that observed previously in some other types of cancer cells. For example, knockdown of Twist in 4T1 and 168FARN mouse breast tumor cells and MDA-MB-435 human melanoma cells does not significantly change cell proliferation and/or their 
Twist is required for viability, migration, invasion and chemoresistance of the

xenograft tumor growth in mice $[4,11,20]$. One recent study even showed that Twist is required for skin tumor initiation and progression in a genedosage-dependent manner. Knockout of Twist in benign tumors reduced cell proliferation and increased apoptosis [21]. These heterogeneous findings suggest that role of Twist in cell proliferation is cell type or sub-tumor type dependent and may be independent from its role in promoting EMT, cell migration and invasion.

It has been reported that Twist expression in breast cancer correlates with lymph node metastasis and poor overall survival [4, 22-24]. According to the molecular profiling data of gene expression, TNBC can be grouped into six subtypes, including two basal-like (BL1 and BL2), an immunomodulatory (IM), a mesenchymal (M), a mesenchymal stem-like (MSL), and a luminal androgen receptor (LAR) subtypes [25]. Twist is mainly expressed in $M$ and MSL subtypes of TNBC and MDAMBLM3 cells studied in the current study belong to the MSL subtype [25]. The current and previous studies have clearly demonstrated that Twist plays a key role in promoting migration, invasion, survival and chemoresistance of breast cancer cells, suggesting That Twist may be a good molecular target to be used for preventing breast cancer metastasis and preventing these cancer cells from development of chemoresistance. Interestingly, a recent study showed that inducible knockout of Twist in adult mice only unlimitedly extended hair growth cycle and had no significant effect on general health conditions, suggesting that Twist may be a tolerable drug target in cancer patients [26]. More interestingly, another recent study

showed that tamoxifen, a widely used drug of endocrine therapy for treating ER -positive breast cancer, can significantly accelerate Twist protein degradation in ER-negative breast cancer cells, leading to inhibition of these cancer cell migration, invasion and metastasis [20]. This finding further supports the notion that inhibition of Twist may be an effective way to control invasive breast cancer. Obviously, more future work needs to be done to develop Twist as a clinical therapeutic target with specific, powerful inhibitors for TNBC treatment.

\section{Acknowledgements}

We thank Li Qin for developing MDA-231LM3.3 cells and Bo Lou, Junjiang $\mathrm{Fu}$ and Dabing $\mathrm{Li}$ for scientific discussion and experimental assistance. This work was partially supported by Sichuan Province grants 2013JY0076 and 2012SZ0073, Luzhou City Grants to the Institute for Cancer Medicine of Sichuan Medical University, and the National Institutes of Health grant CA112403. 


\section{References}

1. Foulkes WD, Smith IE, Reis-Filho JS. Triple-negative breast cancer. N Engl J Med. 2010; 363:

1938-48.

2. Tomao F, Papa A, Zaccarelli E, et al. Triple-negative breast cancer: new perspectives for targeted therapies. Onco Targets Ther. 2015; 8: 177-93.

3. Qin $\mathrm{Q}, \mathrm{Xu} \mathrm{Y}, \mathrm{He} \mathrm{T}$, et al. Normal and disease-related biological functions of Twist1 and underlying molecular mechanisms. Cell Res. 2012; 22: 90-106.

4. Yang J, Mani SA, Donaher JL, et al. Twist, a master regulator of morphogenesis, plays an essential role in tumor metastasis. Cell. 2004; 117: $927-$ 39.

5. Mani SA, Guo W, Liao MJ, et al. The epithelial-mesenchymal transition generates cells with properties of stem cells. Cell. 2008; 133: 704-15.

6. Cheng GZ, Chan J, Wang Q, et al. Twist transcriptionally up-regulates AKT2 in breast cancer cells leading to increased migration, invasion, and resistance to paclitaxel. Cancer Res. 2007; 67:

1979-87.

7. Mironchik Y, Winnard PT, Jr., Vesuna F, et al. Twist overexpression induces in vivo angiogenesis and correlates with chromosomal instability in breast cancer. Cancer Res. 2005; 65:

10801-9.

8. Hu L, Roth JM, Brooks P, et al. Twist is required for thrombin-induced tumor angiogenesis and growth. Cancer Res. 2008; 68: 4296-302.

9. Chen HF, Huang $\mathrm{CH}$, Liu CJ, et al. Twist 1 induces endothelial differentiation of tumour cells

through the Jagged1-KLF4 axis. Nat Commun. 2014; 5: 4697.
10. Hong J, Zhou J, Fu J, et al. Phosphorylation of serine 68 of Twist1 by MAPKs stabilizes Twist1 protein and promotes breast cancer cell invasiveness. Cancer Res. 2011; 71: 3980-90.

11. $\mathrm{Fu} J$, Qin $\mathrm{L}, \mathrm{He} \mathrm{T}$, et al. The TWIST/Mi2/NuRD protein complex and its essential role in cancer metastasis. Cell Res. 2011; 21:275-89.

12. Fu J, Zhang L, He T, et al. TWIST represses estrogen receptor-alpha expression by recruiting the

NuRD protein complex in breast cancer cells. Int J Biol Sci. 2012; 8: 522-32.

13. Yang MH, Hsu DS, Wang HW, et al. Bmil is essential in Twist1-induced epithelial-mesenchymal transition. Nat Cell Biol. 2010; 12: 98292.

14. Shi J, Wang Y, Zeng $\mathrm{L}$, et al. Disrupting the interaction of BRD4 with diacetylated Twist suppresses tumorigenesis in basal-like breast cancer. Cancer Cell. 2014; 25: 210-25.

15. Qin $\mathrm{L}, \mathrm{Wu} \mathrm{YL}$, Toneff $\mathrm{MJ}$, et al. NCOA1 Directly Targets M-CSF1 Expression to Promote

Breast Cancer Metastasis. Cancer Res. 2014; 74: 3477-88.

16. Liu Z, Chen X, Zhou S, et al. The histone $\mathrm{H} 3 \mathrm{~K} 9$ demethylase $\mathrm{Kdm} 3 \mathrm{~b}$ is required for somatic growth and female reproductive function. Int $\mathrm{J}$ Biol Sci. 2015; 11: 494-507.

17. Qin L, Liu Z, Chen $\mathrm{H}$, et al. The steroid receptor coactivator-1 regulates twist expression and promotes breast cancer metastasis. Cancer Res. 2009; 69: 3819-27.

18. Chen X, Qin L, Liu Z, et al. Knockout of SRC-1 and SRC-3 in mice decreases cardiomyocyte proliferation and causes a noncompaction cardiomyopathy phenotype. Int J Biol Sci. 2015; 11: 1056-72. 
19. Gan L, Guo K, Li Y, et al. Up-regulated expression of stathmin may be associated with hepatocarcinogenesis. Oncol Rep. 2010; 23: 1037-43.

20. Ma G, He J, Yu Y, et al. Tamoxifen inhibits ER-negative breast cancer cell invasion and metastasis by accelerating Twist1 degradation. Int J Biol Sci. 2015; 11: 618-28.

21. Beck B, Lapouge G, Rorive S, et al. Different levels of Twist1 regulate skin tumor initiation, stemness, and progression. Cell Stem Cell. 2015; 16: 67-79.

22. Huang J, Ang L, Liu MQ, et al. Serum and tissue expression of gelatinase and Twist in breast cancer. Eur Rev Med Pharmacol Sci. 2014; 18: 2662-9.
23. Zhao $\mathrm{M}, \mathrm{Hu} \mathrm{HG}$, Huang $\mathrm{J}$, et al. Expression and correlation of Twist and gelatinases in breast cancer. Exp Ther Med. 2013; 6: 97-100.

24. $\mathrm{Xu} \mathrm{Y,} \mathrm{Hu} \mathrm{B,} \mathrm{Qin} \mathrm{L}$, et al. SRC-1 and Twist1 expression positively correlates with a poor prognosis in human breast cancer. Int J Biol Sci. 2014; 10: 396-403.

25. Lehmann BD, Bauer JA, Chen X, et al. Identification of human triple-negative breast cancer

subtypes and preclinical models for selection of targeted therapies. J Clin Invest. 2011; 121:

2750-67.

26. $\mathrm{Xu} \mathrm{Y}$, Liao L, Zhou N, et al. Inducible knockout of Twist1 in young and adult mice prolongs hair growth cycle and has mild effects on general health, supporting Twist1 as a preferential cancer target. Am J Pathol. 2013; 183: 1281-92 